\title{
DRL performance of juvenile rats with septal lesions*
}

\author{
ALAN GITTIS and DAVID HOTHERSALL \\ Laboratory of Comparative and Physiological Psychology, The Ohio State University \\ 1314 Kinnear Road, Columbus, Ohio 43212
}

\begin{abstract}
Infant rats received either lesions of the septal nuclei or control operations at 7 days of age. Following weaning at 21 days, the infant rats were trained to leverpress for food. A DRL 20-sec schedule was then used, with the rats being tested in either a conventional operant chamber or one which contained wooden balsa blocks. DRL training continued for 40 daily $60-\mathrm{min}$ sessions. The infant rats successfully leverpressed for food. The presence of the wooden blocks facilitated the development of efficient DRL behavior in the rats with septal lesions, but not in the controls. With training, the juvenile rats with septal lesions trained in conventional chambers showed an improvement in their DRL behavior.
\end{abstract}

In recent years, there has been a revival of interest in the behavioral effects of age-dependent variables (Ambrose, 1969). Many studies have used laboratory rats as Ss, since their offspring are altricial, numerous, and develop rapidly. Weanling rats, between 21 and 35 days old, have been shown to be more active than adults (Furchtgott, Wechkin, \& Dees, 1961; Bronstein, 1972), slower to acquire a passive avoidance habit (Brunner, 1969), and to have poor retention of tasks such as leverpress discrimination (Campbell, Jaynes, \& Misanin, 1968) and active avoidance (Klein \& Spear, 1969). However, few differences have been found between adult and weanling rats in the acquisition of an active avoidance response (Kirby, 1963) and in reversal and probability learning (Roberts, 1966).

Much attention has also been paid to the effects of brain damage early in life and particularly to an animal's ability to functionally recover following brain lesions in infancy (Tsang, 1937a, b; Wetzel, Thompson, Horel, \& Meyer, 1965; Thompson, 1970). Although much of the research has been concerned with cortical recovery, there have been several infant lesion studies involving limbic system structures. Recovery of function in the cat has been studied following amygdalectomy (Kling, 1962, 1965) and hippocampectomy (Isaacson, Nonneman, \& Schmaltz, 1968). In the rat, the effects of infant lesions of the hippocampus (Moorcroft, 1971) and of the septum (Johnson, 1970) have been studied.

In the Johnson (1970) study, several behavioral measures were used to assess recovery of function following septal lesions at 7 days of age. At maturity, the rats were tested in social, conditioned avoidance, and food-reinforced operant situations. Even though 104 days intervened between the time of the lesion and the beginning of testing, these animals showed response characteristics typical of animals given lesions of the septum as adults.

The aim of the present experiment was to continue

*This project was funded in part by the National Institute of Mental Health through Research Grant MH-06211 awarded to Patricia M. Meyer. We sincerely thank Donald R. Meyer and Patricia M. Meyer for their support of this research. this line of investigation of the effects of lesions of the septum early in life. Specifically, our aim was to test such animals shortly after they had sustained lesions in infancy and to continue testing throughout development. The test situation we employed was food-reinforced operant responding with a differential reinforcement of low rate responding (DRL) schedule. We chose this particular schedule because it has been found to differentiate in adult rats between normals and Ss having lesions of the septum (Fried, 1972). Adult rats with lesions of the septum have typically been reported to show a DRL deficit, i.e., an inability to withhold responding and thus an inability to meet the demands of the schedule. It was of interest to determine whether Ss, tested early in life shortly after receiving a lesion of the septum, would demonstrate a similar DRL deficit.

In the design of this experiment, we also included a "mediation" condition, i.e., a condition which had been found in the past to facilitate the development of collateral, mediating behaviors in rats working on time-based schedules of reinforcement (Slonaker \& Hothersall, 1972). Mediation conditions have been found to improve the DRL performance of normal adult animals (Laties, Weiss, \& Weiss, 1969) and adult animals that have lesions of the septum (Hothersall, Alexander, \& Slonaker, 1972). If rats sustained a lesion of the septum early in life and tested while young did indeed show a DRL deficit, it would be interesting to determine if they also would benefit from the opportunity to develop collateral behaviors while working on a time-based schedule of reinforcement.

\section{METHOD}

\section{Subjects}

Male and female pups were selected from eight litters of Long-Evans hooded rats bred in our laboratory. Thirty-one animals completed the experiment, with 19 having received septal lesions ( 9 males, 10 females) and 12 having received control operations ( 8 males, 4 females).

\footnotetext{
Surgery

The surgical technique was only slightly modified from Johnson (1972). Surgery was performed when rats were 7 days
} 


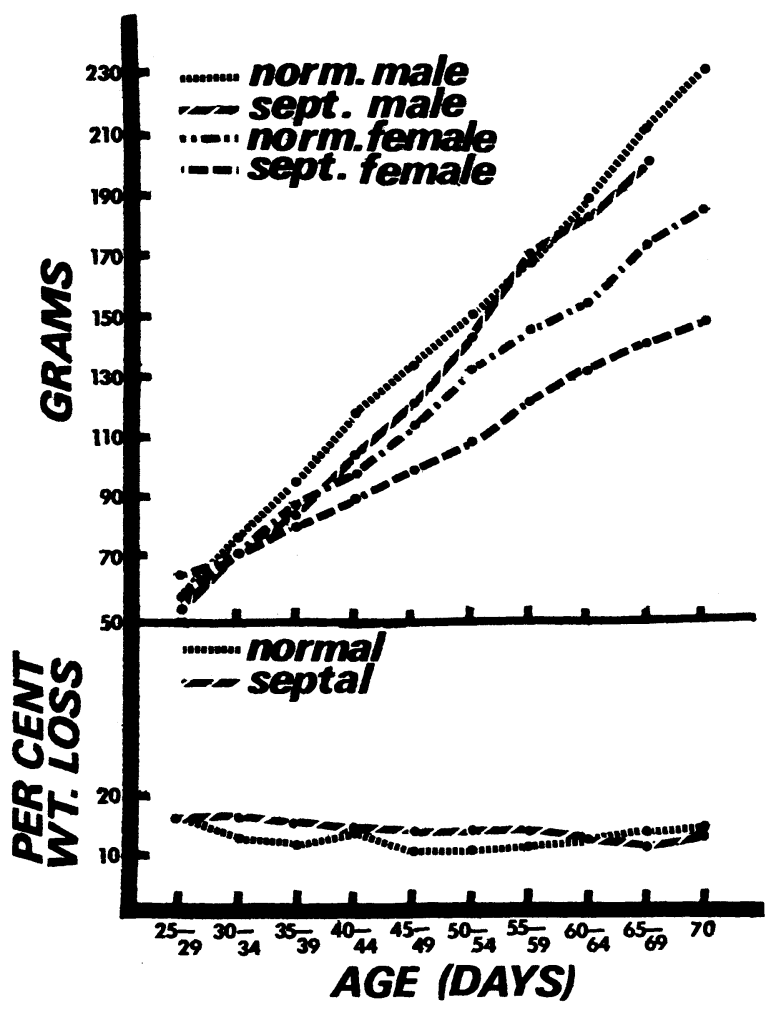

Fig. 1. Top: Weight over training for male and female Ss in lesion and normal groups. Bottom: Percent daily weight loss over training for lesion and normal groups.

of age. The animals were anesthetized by being placed in a jar containing a cotton sponge saturated with methoxyflurane until $1 \mathrm{~min}$ after struggling ceased. The animals were then supported in a clay mold, with the head held horizontally between lambda and bregma. An anterior-posterior incision was made above bregma and a trephine hole was drilled at midline, anterior and tangential to the coronal suture. A steel sewing needle (No. 7 sharp), insulated except for the $.5-\mathrm{mm}$ tip, served as the electrode. The electrode was placed $.4 \mathrm{~mm}$ anterior to the coronal suture and $.5 \mathrm{~mm}$ lateral to the sagittal sinus, and lowered $4.3 \mathrm{~mm}$ from the surface of the cortex. Electrolytic lesions were made bilaterally with a $1.5-\mathrm{mA}$ current delivered for $15 \mathrm{sec}$. The circuit was completed by taping the cathode to the hindlegs and tail, which were painted with electrode cream. After suturing, sulfa powder was applied to the incision, the animals were ear-punched for identification, and then returned to the nest.

Control operates were anesthetized, received skin incisions, and were ear-punched before being returned to the nest.

\section{Procedure}

Animals were raised from birth until weaning in stainless steel maternity cages under continuous light conditions. The mother was removed from the nest for $3-4 \mathrm{~h}$, when the pups were 7 days old, while surgeries were being performed. Also at this time, litters larger than 8 were reduced. Depending on the size of the litter, approximately two-thirds of the pups were given septal lesions and the remainder were given control operations. The latter procedure was used in anticipation of higher mortality rates in the groups with lesions. Animals were weighed at 9, 12, 15 , and 18 days of age to determine whether surgical intervention interfered with growth.

The animals were weaned at 21 days of age, weighed, and placed in individual stainless steel cages. They were fed ad lib for 2 days on a diet of rat pellets, moistened mash (Purina), and apple. At 23 days of age, animals were food deprived and began the 20-h deprivation schedule used for the remainder of the experiment.

\section{Operant Conditioning}

At 24 days of age, an operant shaping procedure began. The rats were placed in conventional operant chambers enclosed in sound-attenuating boxes in rooms apart from the electromechanical programming apparatus. A shaping procedure was used in which, for the first two 30-min training sessions, animals were placed in the operant chambers with the levers removed. Forty-five-milligram pellets (Noyes) were delivered on a noncontingent VI 30-sec schedule. On the third day of shaping, the levers were in place and pellets were made contingent on leverpressing on a CRF schedule. CRF sessions were $1 \mathrm{~h}$ long. Criterion for acquisition of the leverpressing response was the receipt of 75 reinforcements within four sessions.

The day following attainment of criterion, the following DRL training procedure was instituted: 10 reinforcements were given on DRL $10 \mathrm{sec}, 10$ reinforcements on DRL $15 \mathrm{sec}$, and the rats were then allowed to respond for the remainder of the 60 -min session on a DRL 20 -sec schedule.

The following day, the first of 40 1-h DRL 20-sec training sessions was given. The rats were then between 28 and 32 days old. The Ss were run daily. Both septal and normal rats were randomly assigned to either the mediation or the nonmediation groups. In the mediation condition, $3 \times 2 \times 1 \frac{1}{2}$ in. balsa wood blocks were mounted 2 in. apart on the wall opposite the lever in the operant chamber. Any rat in a mediation group that did not consistently chew the wood after 10 sessions was removed from the study. Six normal rats and one rat with a septal lesion were eliminated. The rats in the nonmediation conditions did not have the wooden blocks in the operant chambers. Responses and reinforcements were recorded at the end of eachday's session. In addition, the amount of balsa wood chewed was collected and weighed each day. After each daily session, the rats were returned to their home cages and given access to rat pellets and apple slices for $3 \mathrm{~h}$. Eleven nonmediated septal rats $(5$ males, 6 females), 8 mediated septal rats (4 males, 4 females), 7 nonmediated normals ( 4 males, 3 females), and 5 nonmediated normals (4 males, 1 female) completed 40 sessions of DRL 20 sec.

At the beginning of the study, animals were weighed every few days to assess whether they were gaining weight steadily. We then became increasingly interested in this data and began to weigh the animals twice a day, before placing them in the operant chamber and after deprivation. Complete weight data was collected on 4 septals ( 2 males, 2 females) and 4 normals ( 3 males, 1 female), and percent daily weight loss through the course of DRL training was calculated.

When training was completed, the rats with lesions of the septum were given a lethal dose of sodium pentobarbital and perfused intracardially with physiological saline, followed by $10 \%$ Formalin. The brains were then embedded in celloiden and serially sectioned at 30 microns. Every fifth section through the septum was mounted on glass slides and stained with cresyl violet.

\section{RESULTS}

Though the juvenile rats were maintained on a deprivation schedule from 23 days of age until approximately 70 days of age, both septal and normal rats remained healthy and gained weight consistently (Fig. 1). Since the number of consistently weighed animals was small, weight differences between normal and septal rats were not statistically analyzed. In the course of training, the percent body weight loss from 
day to day varied little, ranging across all groups and both sexes from approximately $16 \%$ at the beginning of training to $14 \%$ at the conclusion of the study.

Rats with septal lesions tended to shape more quickly than normals. Seventy-four percent of the septal animals had attained the shaping criterion of receipt of 75 reinforcements by the second day of training, whereas only $42 \%$ of the normals had done so. There was no systematic difference due to sex. Five septals out of a total of 24 and 2 normals out of a total of 16 failed to attain the shaping criterion.

Young rats in operant chambers emit unique and unconventional responses. Frequently, they stood on their hindlegs to reach the lever and often they had to jump to exert enough pressure to depress it. Despite the relatively greater exertion required of juvenile rats, the number of responses emitted in a session was highly comparable to that of adult animals. At the beginning of the DRL training, normal nonmediated adult rats, trained in our laboratory, have been found to make, on the average, 192 responses in a 60 -min session, while adult rats with septal lesions made 388 responses (Slonaker, 1971). During the comparable period in the present experiment, normal juvenile rats averaged 215 leverpresses in a 60-min session, while septal juveniles emitted 431 responses.

Three measures were taken as indices of the animal's DRL performance: responses per session, reinforcements per session, and the efficiency ratio (reinforcements/responses) (Brady \& Conrad, 1960). Data for daily sessions were grouped into 10 blocks of four sessions for analysis. A separate four-factor analysis of variance (Lesion by Mediation by Sex by Days, with repeated measures on days) was computed for all three measures. Correlations between the measures were also computed. Responses were highly negatively correlated with efficiency $(-.76)$, and reinforcements were highly positively correlated (.70). The correlation between responses and reinforcements was -.51 . The magnitude of the differences found in each analysis was highly comparable, thus the statistical results reported will refer to the analysis of the efficiency ratios.

The results of this experiment are shown in Fig. 2. Compared to the normal Ss, the rats with lesions of the septum, particularly at the beginning of training, showed a higher response rate, received a smaller number of reinforcements, and had a lower DRL efficiency. The statistical analysis showed a significant main effect of lesion $(F=158.9, \mathrm{df}=1 / 230, \mathrm{p}<.001)$. Figure 2 also indicates that the presence of the wood blocks improved the performance of the rats with septal lesions, with this effect being stronger at the beginning of training. Statistically, the main effect of mediation was significant $(\mathrm{F}=10.5, \mathrm{df}=1 / 230, \mathrm{p}<.001)$, as was the interaction between the lesion and mediation factors $(\mathrm{F}=10.0, \mathrm{df}=$ $1 / 230, p<.002)$. Post hoc comparisons using the Scheffé test showed that allowing access to the wood blocks significantly improved the performance of rats

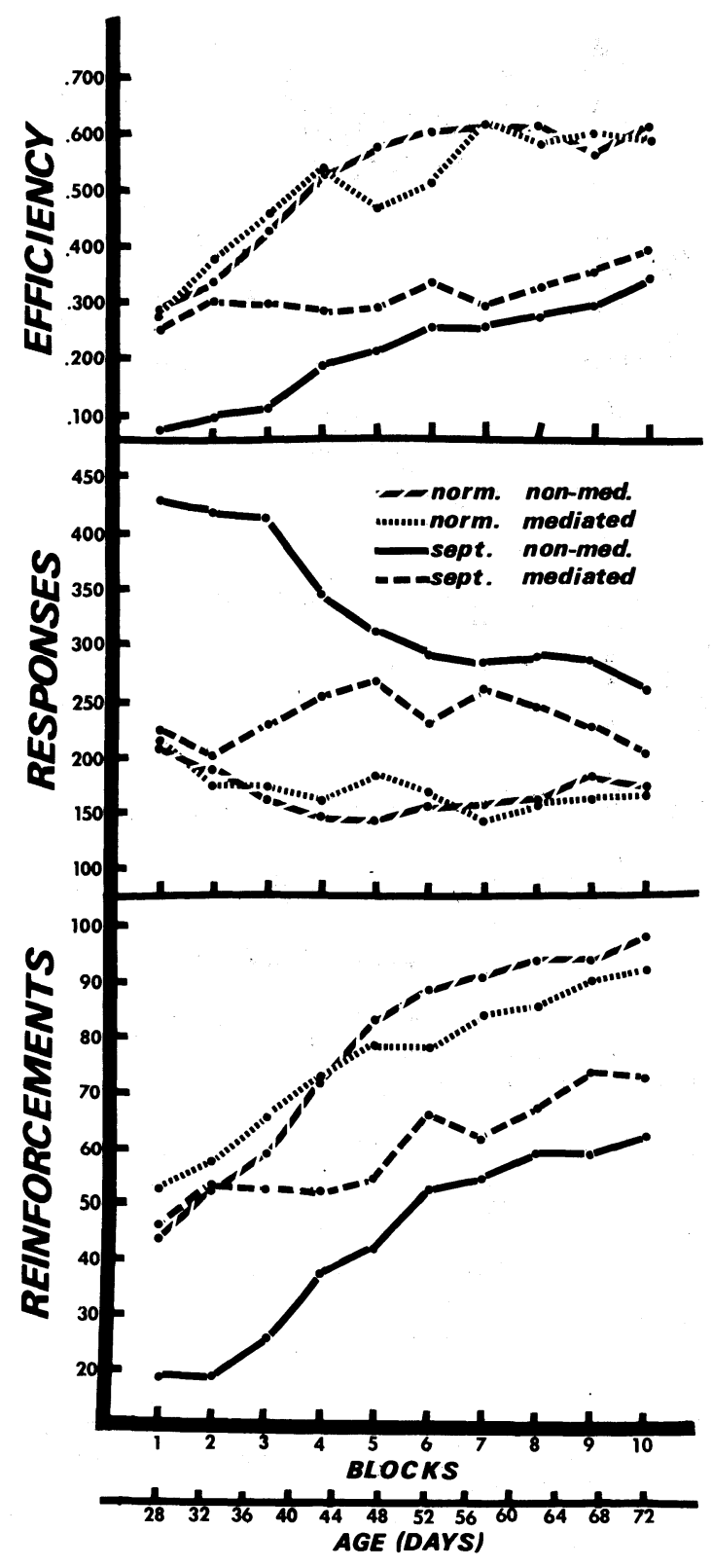

Fig. 2. Performance over blocks of four daily sessions for normal and lesioned $S s$ in both mediation and nonmediation conditions. Top: Efficiency ratio. Middle: Responses. Bottom: Reinforcements.

with septal lesions but not the performance of normal Ss $(s=.065, \mathrm{df}=1 / 230, \mathrm{p}=.05)$. The male rats with septal lesions chewed more wood than any other group, and the normal males chewed more than the females with septal lesions. The correlations between amount of wood chewed and DRL efficiency were .54 for the normal rats and .33 for the rats with septal lesions.

During training, the DRL efficiency of both groups of normal rats improved, a not unexpected result, but somewhat surprisingly both groups of rats with septal lesions also showed some improvement in their DRL performance as training continued. Statistically, the 

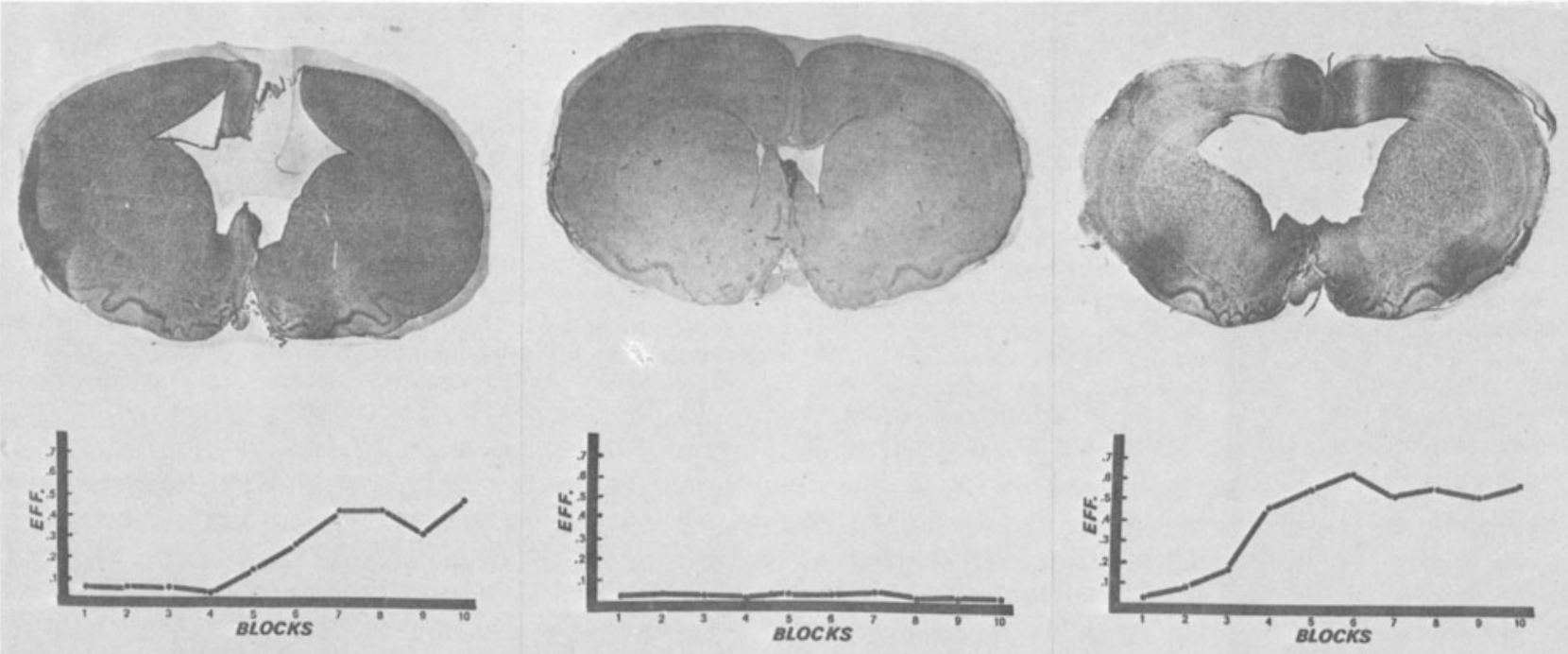

Fig. 3. Photographs of cresyl-violet-stained sections showing area of maximal septal damage in three nonmediated Ss with their efficiency ratios over training.

main effect of days was significant $(F=6.8, \mathrm{df}=9 / 230$, $\mathrm{p}<.001)$. However, the Days by Lesion interaction was not significant $(F=1.1$, df $=9 / 230)$, indicating that both the normal rats and those with lesions of the septum showed improvement on DRL as training continued.

Figure 3 shows representative sections through the area of maximal septal damage of three nonmediated juveniles together with their efficiency ratios over training. Histological analysis revealed that in all Ss receiving lesions, some septal damage was present, but the extent and locus of the lesion was highly variable. Often there was cortical, callosal, and caudate damage. Posteriorly, there was often damage to the fornix, but usually unilaterally. No prediction as to the animal's performance over the course of training could be made based upon analysis of the extent of damage to the septal nuclei and invasion into adjacent structures. Dramatic improvement in DRL performance was noted in animals with nearly complete destruction of the medial and lateral septal nuclei.

\section{DISCUSSION}

This study has shown the feasibility of testing prepubertal rats in an operant setting. Despite the necessity in such research of maintaining juvenile rats on a deprivation schedule, they remained healthy thoughout the experiment. However, these Ss did not grow at the same rate as untreated rats, nor did they attain comparable adult body weights.

Campbell (1967) has discussed the difficulties involved in developing appetitive tasks to be used with young animals, and has suggested that comparisons of the behavior of animals of different ages should only be made with great caution. Establishing comparable motivational levels in young and adult animals is a particularly difficult problem. In this experiment, we used percent body weight loss from day to day as an index of motivation. This measure varied little throughout training, suggesting that motivation level remained relatively constant.

Slonaker and Hothersall (1972) found that the presence of wooden blocks, made from soft pine, increased DRL efficiency in both normal adult rats and adult rats with septal lesions, apparently by affording the rats an opportunity to develop collateral behavior chains. In this experiment, wooden blocks, made from balsa, increased the DRL efficiency of juvenile rats with septal lesions but not the efficiency of normal juvenile rats. Since the balsa blocks are much softer, perhaps hardness of the blocks relates to their usefulness in the development of collateral behavior chains, harder blocks taking more time to chew. Another possibility is that juvenile rats, being more active (Furchtgott, Wechkin, \& Dees, 1961; Bronstein, 1972), are more adept at forming spontaneous collateral behavior chains, with the result that the wooden blocks were of less value to them. However, why the wooden blocks facilitated the performance of the rats with septal lesions but not that of the normal rats is an unresolved question.

The most surprising result of this experiment was the clear decrease in response rate and consequent improvement in DRL efficiency shown by the juvenile rats which received septal lesions in infancy. One animal with a septal lesion, for example, attained an efficiency 
ratio of .75 at the end of training. Such high DRL efficiency levels are not typical of rats that receive septal lesions as adults (Burkett \& Bunnell, 1966; Ellen \& Aitken, 1971). Several explanations of this difference between the consequences of septal damage in young and adult animals can be proposed: nervous system plasticity, increased learning ability of the young animals, response characteristics of the young rats, or characteristics of the testing situation. Based on the research of Johnson (1972), the hypothesis that nervous system plasticity is responsible for the recovery is quite unlikely. Animals lesioned in infancy and tested as adults show behaviors typical of animals lesioned as adults. The hypothesis of increased learning ability in the young animals is probably also inadequate as an explanation of these results.

It is more likely that the response characteristics of the animals coupled with the characteristics of the testing situation, contributed to the results. Younger rats are more active than adult rats (Furchtgott, Wechkin, \& Dees, 1961; Bronstein, 1972), and prepubertally, septal rats are more active than normal controls (Johnson, 1972). This heightened activity, occurring in an apparatus in which there is proportionally more room for locomotion in a juvenile as compared to an adult rat, may have facilitated the development of collateral behavior chains, and thus improved DRL efficiency.

Operant analysis of juvenile animals seems quite feasible, and differences in response characteristics between juvenile and adult septal animals do appear to exist. Problems of apparatus size, activity level, and motivational factors are several directions that further research can take.

\section{REFERENCES}

Ambrose, A. (Ed.) Stimulation in early infancy. New York: Academic Press, 1969.

Brady, J. V., \& Conrad, D. G. Some effects of brain stimulation on timing behavior. Journal of the Experimental Analysis of Behavior, 1960, 3, 93-106.

Bronstein, P. M. Open-field behavior of the rat as a function of age: Cross-sectional and longitudinal investigations. Journal of Comparative \& Physiological Psychology, 1972, 80, 335-341.

Brunner, R. L. Age difference in one-trial passive avoidance learning. Psy chonomic Science, 1969, 14, 134.

Burkett, E. E., \& Bunnell, B. N. Septal lesions and the retention of DRL performance in the rat. Journal of Comparative \& Physiological Psychology, 1969, 67, 344-351.
Campbell, B. A. Developmental studies of learning and motivation in infraprimate mammals. In $\mathbf{H}$. W. Stevenson, $\mathrm{E}$. H. Hess, and H. L. Rhêingold (Eds.), Early behavior: Comparative and developmental approaches. New York: Wiley, 1967.

Campbell, B. A., Jaynes, J. R., \& Misanin, J. Retention of a light-dark discrimination in rats of different ages. Journal of Comparative \& Physiological Psychology, 1968, 66, 467-472.

Ellen, P., \& Aitken, W. C. Absence of temporal discrimination following septal lesions. Psychonomic Science, 1971, 22, 129-131.

Fried, P. A. Septum and behavior: A review. Psychological Bulletin, 1972, 78, 292-310.

Furchtgott, E., Wechkin, S., \& Dees, J. W. Open-field exploration as a function of age. Journal of Comparative \& Physiological Psychology, 1961, 54, 386-388.

Hothersall, D., Alexander, D., \& Slonaker, R. The DRL deficit of rats with septal lesions: Effects of extended training in a mediated environment. Psychonomic Science, 1972, 29, 34-36.

Isaacson, R. L., Nonneman, A. J., \& Schmaltz, L. W. Behavioral and anatomical sequelae of the infant limbic system. In $R$. $L^{\prime}$ Isaacson (Ed.), The neuropsychology of development. New York: Wiley, 1968. Pp. 41-78.

Johnson, D. A. Developmental aspects of recovery of function following septal lesions in the infant rat. Journal of Comparative \& Physiological Psychology, 1972, 78, 331-348.

Kirby, R. H. Acquisition, extinction, and retention of an avoidance response in rats as a function of age. Journal of Comparative \& Physiological Psychology, 1963, 56, 158-162.

Klein, S. B., \& Spear, N. E. Influence of age in short-term retention of active avoidance learning in rats. Journal of Comparative \& Physiological Psychology, 1969, 69, 583-589.

Kling, A. Amygdalectomy in the kitten. Science, 1962, 137, 429-430.

Kling, A. Behavioral and somatic development following lesions of the amygdala in the cat. Journal of Psychiatric Research 1965, 3, 263-273.

Laties, V. G., Weiss, B., \& Weiss, A. B. Further observations on overt "mediating" behavior and the discrimination of time. Journal of the Experimental Analysis of Behavior, 1969, 12, 43-57.

Moorcroft, W. H. Ontogeny of forebrain inhibition of behavioral arousal in the rat. Brain Research, 1971, 35, 513-522.

Roberts, W. A. Learning and motivation in the immature rat. American Journal of Psychology, 1966, 79, 3-23.

Slonaker, R. L. Collateral behaviors and the DRL deficit of rats with septal lesions. Unpublished master's thesis, The Ohio State University, 1971.

Slonaker, R. L., \& Hothersall, D. Collateral behaviors and the DRL deficit of rats with septal lesions. Journal of Comparative \& Physiological Psychology, 1972, 80, 91-96.

Thompson, V. E. Visual decortication in infancy in rats. Journal of Comparative \& Physiological Psychology, 1970, 72, 444-451.

Tsang, $Y$. C. Maze learning in rats hemidecorticated in infancy. Journal of Comparative Psychology, 1937a, 24, 221-254.

Tsang, Y. C. Visual sensitivity in rats deprived of visual cortex in infancy. Journal of Comparative Psychology, 1937b, 24, 255-262.

Wetzel, A. B., Thompson, V. E., Horel, J. A., \& Meyer, P. M. Some consequences of the perinatal lesions of the visual cortex in the cat. Psychonomic Science, 1965, 3, 381-382.

(Received for publication November 1, 1973; revision received December 11,1973 .) 